\title{
28 Research Soure \\ Validation of Fatty Liver Index as a Marker for Metabolic Dysfunction-Associated Fatty Liver Disease
}

\author{
A lum Han ( $\square$ qibosarang@naver.com ) \\ Wonkwang University Medical Center \\ Youngjon Kim
}

Department of Medical Education, Wonkwang University School of Medicine

\section{Research Article}

Keywords: Fatty liver index, Metabolic dysfunction associated fatty liver disease, Computed tomography, nonalcoholic fatty liver disease, fatty liver, obesity, body mass index, alanine aminotransferase, gammaglutamyl transferase, highly sensitive C-reactive protein

Posted Date: July 14th, 2021

DOI: https://doi.org/10.21203/rs.3.rs-494412/v2

License: (a) (i) This work is licensed under a Creative Commons Attribution 4.0 International License. Read Full License 


\section{Abstract}

Background: Metabolic dysfunction-associated fatty liver disease (MAFLD) is a new nomenclature for non-alcoholic fatty liver. Fatty liver associated with metabolic dysfunction is increasing with obesity and has become a serious socioeconomic problem. Non-invasive testing to confirm MAFLD, such as the fatty liver index (FLI), can be used as an alternative method for diagnosing steatosis when imaging modalities are not available. To date, few studies have examined the effectiveness and validity of FLI for diagnosing MAFLD. Therefore, this study analyzed the effectiveness and validity of FLI for diagnosing MAFLD.

Methods: The medical records of men and women aged 19 years or older who underwent abdominal computed tomography (CT) examination at the University Hospital Health Promotion Center from March 2012 to October 2019 were reviewed retrospectively. A comparative analysis between non-continuous variables was performed using the chi-squared test. The area under receiver operating characteristic (AUROC) curve was used to verify the effectiveness of FLI as a predictive index for MAFLD.

Results: Analysis of the association between MAFLD and abdominal CT revealed that the sensitivity and specificity of FLI for diagnosing MAFLD were 0.712 and 0.713 , respectively. The AUROC of FLI for the prediction of MAFLD was 0.776 .

Conclusion: Our study verified the accuracy of FLI for predicting MAFLD using CT. Additionally, FLI can be used as a simple and cost-effective tool for screening MAFLD in clinical settings

\section{Introduction}

Metabolic dysfunction-associated fatty liver disease (MAFLD), previously termed nonalcoholic fatty liver disease (NAFLD), is a new nomenclature and concept for nonalcoholic fatty liver [1]. Fatty liver associated with metabolic dysfunction is common. The prevalence of NAFLD is increasing with obesity and has become a serious socioeconomic problem [2]. Inaccuracies in the terms and definitions of the heterogeneous etiology of metabolic fatty liver require reevaluation [3]. Clinically, there was a need for a concept that could more accurately reflect management design and pathological mechanisms [2-4]. Experts agree that NAFLD, widely used and utilized to date, does not reflect metabolic dysfunction. This is because NAFLD could only be defined without consuming alcohol and no other metabolic disorders [4]. NAFLD is currently the most common cause of chronic liver disease and is one of the leading causes of cirrhosis around the world, so it is attracting attention and needs to be re conceptualized [5]. The diagnosis of NAFLD requires evidence of hepatic steatosis, the exclusion of excessive alcohol consumption, and the exclusion of other liver diseases. Because of this exclusion, it was perceived that the diagnostic criteria were rather insufficient. Against this background, a group of international experts recently proposed to rename NAFLD to MAFLD. The new name MAFLD well reflects its role in fatty liver as well as diseases of type 2 diabetes, hypertension, dyslipidemia and obesity. In other words, even if chronic metabolic diseases exist at the same time, the diagnosis can be made, and the effects of chronic viral hepatitis and alcohol on the liver were considered [6]. This led to a better explanation of the role of 
alcohol, viral liver disease, and metabolic dysfunction in fatty liver. The prognosis and condition of fatty liver can now be better recognized [6].

The change to MAFLD is not just a nomenclature change; it is difficult to classify a liver disease as MAFLD even if it meets the criteria for NAFLD. Even if hepatic steatosis is present, it can be classified as MAFLD only if there are elements of metabolic dysfunction that meet the criteria. The name was changed from NAFLD to MAFLD with some difficulties. Difficulties in the development of therapeutic drugs for NAFLD and biomarkers for the identification of NAFLD, specifically, non-invasive testing to confirm NAFLD is a major focus in this area. One of the most important barriers to identifying reliable biomarkers is disease heterogeneity and the dynamic nature of histopathology, which is no longer clear with the proposed name change. As the disease phenotype becomes clearer, biomarker development is accelerated [3]. Abdominal ultrasonography is usually sufficient to detect hepatic steatosis and is the recommended primary diagnostic tool for MAFLD imaging [6]. Scores such as the fatty liver index (FLI), which can be computed with serum biomarkers, can be used as an alternative method for diagnosing steatosis when imaging modalities are not available or viable, such as in large-scale epidemiological studies [7]. FLI predicted NAFLD using body mass index (BMI), waist circumference (WC), and triglyceride (TG) and $y$-glutamyl-transferase ( $\mathrm{Y}-\mathrm{GT}$ ) levels. The statistical significance of FLI was demonstrated in a large-scale study using ultrasound to diagnose NAFLD [7]. In several European studies, FLI has shown excellent results in predicting fatty liver $[8,9]$. In studies conducted in Asia, where race and environment are different from those in Europe, studies have shown good area under the receiver operating characteristics (AUROC) curve, and FLI was statistically significant $(P<0.0001)$ in predicting fatty liver $[10,11]$. However, to date, few studies have examined the effectiveness and validity of FLI for diagnosing MAFLD. Therefore, this study analyzed the effectiveness and validity of FLI for diagnosing the newly defined MAFLD. Ultrasound is most commonly used when diagnosing fatty liver; however, computed tomography (CT) of the abdomen has more reliable advantages, including reproducibility, objectivity, and specificity [12]. Therefore, we used CT instead of ultrasound to check the accuracy of FLI for diagnosing fatty liver.

\section{Methods}

\section{Participants}

The medical records of men and women aged 19 years or older who underwent abdominal CT examination at the University Hospital Health Promotion Center from March 2012 to October 2019 were retrospectively reviewed. Patients with incomplete records, acute infectious diseases, cancer, and findings suggestive of cancer were excluded, and 1,059 participants were included in the final analysis. Among them, 852 participants who had all the records of the indicators for calculating the FLI were analyzed. This study followed the ethical standards established in the Declaration of Helsinki. Consent was not required from all participants. We only reviewed patient charts for this study, we pledged that we would not use the information for anything other than research purposes. Also we reviewed the chart, which 
provided only the number of participants instead of their names. This was approved by the institutional review board of the Wonkwang University Hospital (ARB Approval No. 2020-06-002-002).

MAFLD diagnosis

MAFLD was diagnosed based on one of the following criteria:

1. Overweight or obesity (defined as BMI of $\geq 23 \mathrm{~kg} / \mathrm{m}^{2}$ ) and fatty liver on a CT scan.

2. Lean/normal weight (defined as BMI of $<23 \mathrm{~kg} / \mathrm{m}^{2}$ ) and fatty liver on a CT scan with at least two metabolic risk abnormalities:

- WC of $\geq 102 / 88 \mathrm{~cm}$ in Caucasian men and women (or $\geq 90 / 80 \mathrm{~cm}$ in Asian men and women).

- Blood pressure $\geq 130 / 85 \mathrm{mmHg}$ or receiving specific drug treatment.

- Plasma triglycerides level of $\geq 150 \mathrm{mg} / \mathrm{dL}(\geq 1.70 \mathrm{mmol} / \mathrm{L})$ or receiving specific drug treatment.

- Plasma HDL cholesterol level of $<40 \mathrm{mg} / \mathrm{dl}(<1.0 \mathrm{mmol} / \mathrm{L})$ for men and $<50 \mathrm{mg} / \mathrm{dL}(<1.3 \mathrm{mmol} / \mathrm{L})$ for women or receiving specific drug treatment.

- Prediabetes (i.e., fasting glucose levels of $100-125 \mathrm{mg} / \mathrm{dL}$ [5.6-6.9 mmol/L], 2-h post-load glucose levels of $140-199 \mathrm{mg} / \mathrm{dL}$ [7.8-11.0 mmol] or hemoglobin $(\mathrm{Hb})$ A1c level of $5.7-6.4 \%$ [39-47 $\mathrm{mmol} / \mathrm{mol}])$

- Homeostasis model assessment of insulin resistance scores of $\geq 2.5$.

- Plasma high-sensitivity C-reactive protein levels of $>2 \mathrm{mg} / \mathrm{L}$.

3. Diabetes mellitus (according to widely accepted international criteria) and fatty liver on a CT scan.

SOMATOM definition (Siemens Medical Solutions, Forchheim, Germany) was used for CT scans of the abdominal pelvis, and image readings were performed by a radiologist. To avoid examiner bias, all data were reconfirmed by a medical imaging specialist who was blinded to the patient's characteristics, medical history, and research objectives. Fatty liver was diagnosed when the liver attenuation value was $<40$ Hounsfield units $(\mathrm{HU})$ or $<10 \mathrm{HU}$ compared to that of the spleen.

WC measured by the measurer and examinee using a waist tape was prone to large errors. Therefore, WC was measured according to the level recommended by the World Health Organization at the mid (half) point between the lowest rib and iliac ridge on $\mathrm{CT}$ images.

Anthropometric assessment and blood test

The height and weight were measured using an automatic height scale. BMI was used to check for obesity and overweight. BMI was calculated by dividing body weight by height squared $\left(\mathrm{kg} / \mathrm{m}^{2}\right)$. According to the Asian criteria, a BMI of $\geq 23$ was defined as overweight, and a BMI of $\geq 25$ was defined as obesity. After keeping the blood pressure stable for at least 10 mins, an automatic 
sphygmomanometer was used to measure the blood pressure, and the average of the two measurements was recorded.

After fasting for $>8 \mathrm{~h}$, blood was collected from a vein and was immediately sent to the Neodine Lab for liver function tests, complete blood cell count, blood lipid level, blood sugar level, and serum tests. Fasting blood sugar (FBS), insulin, total cholesterol (TC), triglycerides (TG), high-density lipoprotein cholesterol (HDL-C), low-density lipoprotein cholesterol (LDL-C), alanine aminotransferase (ALT), aspartate amino transferase (AST), gamma-glutamyl transpeptidase (GGT), uric acid (UA), creatinine ( $\mathrm{Cr}$ ), highly sensitive C-reactive protein (hs-CRP), white blood cell (WBC), hemoglobin, and vitamin D concentrations were measured.

Clinical and lifestyle assessments

The experts at the health screening center used self-questionnaires to examine participants' medical history and lifestyle. Participants who were diagnosed with type 2 diabetes or receiving drugs were recorded. Participants were classified as smokers and non-smokers, and those who smoked continuously for the past two years were classified as smokers. If they consumed a meaningful amount of alcohol at least once a week, alcohol consumption was marked as 'yes'.

FLI calculation

$\mathrm{FLI}=(\mathrm{e}(0.953 \times \ln (\mathrm{TG})+0.139 \times \mathrm{BMI}+0.718 \times \ln (\mathrm{GGT})+0.053 \times \mathrm{WC}-15.745)) /(1+\mathrm{e}(0.953 \times \ln (\mathrm{TG})+$ $0.139 \times \mathrm{BMI}+0.718 \times \ln (\mathrm{GGT})+0.053 \times \mathrm{WC}-15.745)) \times 100$

$T G$, triglyceride $(\mathrm{mg} / \mathrm{dL})$; GGT, $\mathrm{Y}$-glutamyl transferase $(\mathrm{U} / \mathrm{L})$; $\mathrm{WC}$, waist circumference $(\mathrm{cm})$. BMI was calculated by dividing body weight by the square of height $\left(\mathrm{kg} / \mathrm{m}^{2}\right)$ [13].

Statistical analyses

SPSS for Windows (version 26.0; SPSS Inc., Chicago, IL, USA) was used for all statistical analyses. A comparative analysis between non-continuous variables was performed using the chi-squared test.

The AUROC curve was used to verify the effectiveness of FLI as a predictive index for MAFLD. The method developed by DeLong et al. was used to compare the FLI and AUROC.

FLI's ability to distinguish between participants with and without MAFLD was assessed using the ROC curve. The sensitivity of the infinite determination threshold of FLI was expressed as a false positive rate, and the relevant area under the curve (AUC) was calculated. The lower limit of the AUC was considered as 0.5 , and the area $>0.5$ demonstrates the effectiveness of $F L I$ in distinguishing patients with and without MAFLD. The optimal cutoff point for FLI was determined using the maximum value of Youden's $J$ statistic [max $(\mathrm{J}=$ sensitivity + specificity -1$)]$. The value of FLI, which corresponds to the maximum value of the Youden index, was considered the best reference point for FLI [8]. 


\section{Results}

General patient characteristics with or without MAFLD

The total number of participants meeting the criteria was 852, with 574 without MAFLD and 278 with MAFLD. The clinical and biochemical characteristics of the MAFLD and normal groups are described in Table 1. Among the participants with MAFLD, 209 were men (75.2\%), and 69 were women (24.8\%); $66.2 \%$ were non-smokers; $57 \%$ had an FLI of $\geq 60$, and $43 \%$ had an FLI of $<30$. In the MAFLD group, BMI, systolic blood pressure, diastolic blood pressure, WC, FBS, TG, AST, ALT, GGT, insulin, and UA/Cr levels were higher than the control group, and HDL level was lower in the MAFLD group. Particularly, the FLI was significantly higher in the MAFLD group. There was no significant difference in vitamin D levels between the two groups. 
Table 1

Characteristics of the subject according to MAFLD

\begin{tabular}{|c|c|c|c|c|c|}
\hline \multirow{2}{*}{\multicolumn{2}{|c|}{$\begin{array}{l}\text { Total } \\
\text { subjects(= 852) }\end{array}$}} & \multicolumn{2}{|l|}{ MAFLD } & \multirow[t]{2}{*}{ p-value } & \multirow[t]{4}{*}{ AUROC(95\% Cl) } \\
\hline & & \multirow{2}{*}{$\begin{array}{l}\text { NO = 574 } \\
300(52.3)\end{array}$} & \multirow{2}{*}{$\begin{array}{l}\text { YES = 278 } \\
209(75.2)\end{array}$} & & \\
\hline Sex & Male & & & \multirow[t]{2}{*}{$<0.0001$} & \\
\hline & Female & $274(47.7)$ & $69(24.8)$ & & \\
\hline \multirow[t]{2}{*}{ Alcohol } & $\mathrm{N}$ & $360(62.7)$ & $160(57.6)$ & \multirow[t]{2}{*}{0.147} & \\
\hline & $\mathrm{Y}$ & 214(37.3) & $118(42.4)$ & & \\
\hline \multirow[t]{2}{*}{ Smoking } & $\mathrm{N}$ & 446(77.7) & 184(66.2) & \multirow[t]{2}{*}{$<0.0001$} & \\
\hline & $\mathrm{Y}$ & 128(22.3) & $94(33.8)$ & & \\
\hline \multirow[t]{2}{*}{ FLI } & $30<$ & 407(89.8) & $80(43.0)$ & \multirow[t]{2}{*}{$<0.0001$} & \\
\hline & $\geq 60$ & $46(10.2)$ & $106(57.0)$ & & \\
\hline \multicolumn{2}{|l|}{ Age (yr) } & $52.44 \pm 10.20$ & $52.97 \pm 9.31$ & \multicolumn{2}{|l|}{0.462} \\
\hline \multicolumn{2}{|l|}{ BMI } & $23.63 \pm 2.84$ & $26.64 \pm 3.12$ & \multicolumn{2}{|l|}{$<0.0001$} \\
\hline \multicolumn{2}{|c|}{$\mathrm{SBP}(\mathrm{mmHg})$} & $121.19 \pm 12.59$ & $126.15 \pm 11.77$ & \multicolumn{2}{|l|}{$<0.0001$} \\
\hline \multicolumn{2}{|c|}{$\mathrm{DBP}(\mathrm{mmHg})$} & $74.63 \pm 9.56$ & $78.84 \pm 10.02$ & \multicolumn{2}{|l|}{$<0.0001$} \\
\hline \multicolumn{2}{|l|}{ WC (cm) } & $82.34 \pm 8.07$ & $89.23 \pm 9.17$ & \multicolumn{2}{|l|}{$<0.0001$} \\
\hline \multicolumn{2}{|c|}{ FBS (mg/dL) } & $92.3 \pm 23.38$ & $99.93 \pm 26.27$ & \multicolumn{2}{|l|}{$<0.0001$} \\
\hline \multicolumn{2}{|c|}{ TG (mg/dL) } & $98.8 \pm 64.16$ & $150.91 \pm 143.04$ & \multicolumn{2}{|l|}{$<0.0001$} \\
\hline \multicolumn{2}{|c|}{ HDL-C (mg/dL) } & $56.74 \pm 12.9$ & $52.4 \pm 13.72$ & \multicolumn{2}{|l|}{$<0.0001$} \\
\hline \multicolumn{2}{|c|}{ LDL-C (mg/dL) } & $119.53 \pm 32.72$ & $124.16 \pm 38.03$ & \multicolumn{2}{|l|}{0.082} \\
\hline \multicolumn{2}{|c|}{ AST (IU/L) } & $30.82 \pm 21.94$ & $37.66 \pm 38.42$ & 0.001 & \\
\hline ALT (IU/L) & & $27.52 \pm 24.32$ & $40.15 \pm 29.71$ & $<0.0001$ & $0.701(0.657-0.745)$ \\
\hline GGT (IU/L & & $36.76 \pm 66.86$ & $72.79 \pm 215.35$ & 0.007 & $0.668(0.622-0.714)$ \\
\hline Uric acid/ & eatinine & $6.29 \pm 1.75$ & $6.61 \pm 1.56$ & 0.011 & \\
\hline hsCRP (m & & $1.65 \pm 3.93$ & $2.03 \pm 3.61$ & 0.216 & $0.620(0.572-0.668)$ \\
\hline $\mathrm{TC}(\mathrm{mg} / \mathrm{d}$ & & $199.95 \pm 35.45$ & $205.11 \pm 44.30$ & 0.091 & \\
\hline WBC $(10 *$ & $\mu \mathrm{L})$ & $6019.63 \pm 1735.57$ & $6722.82 \pm 1950.21$ & $<0.0001$ & \\
\hline $\mathrm{Hb}(\mathrm{g} / \mathrm{dL})$ & & $14.23 \pm 1.50$ & $15.34 \pm 1.50$ & $<0.0001$ & $0.682(0.635-0.729)$ \\
\hline Insulin $(\mu$ l & (mL) & $4.41 \pm 3.02$ & $7.76 \pm 6.46$ & $<0.0001$ & $0.717(0.672-0.761)$ \\
\hline
\end{tabular}




\begin{tabular}{|c|c|c|c|c|}
\hline \multirow{2}{*}{$\begin{array}{l}\text { Total } \\
\text { subjects(= 852) }\end{array}$} & \multicolumn{2}{|l|}{ MAFLD } & \multirow[t]{2}{*}{ p-value } & \multirow[t]{2}{*}{ AUROC(95\% Cl) } \\
\hline & NO $=574$ & YES $=278$ & & \\
\hline $\operatorname{VitD}(\mathrm{ng} / \mathrm{mL})$ & $18.99 \pm 8.89$ & $19.27 \pm 7.86$ & 0.688 & \\
\hline FLI & $23.1 \pm 20.75$ & $48.63 \pm 25.5$ & $<0.0001$ & $0.776(0.737-0.816)$ \\
\hline \multicolumn{5}{|c|}{$\begin{array}{l}\text { Abbreviations: MAFLD, metabolic associated fatty liver disease; SBP, Systolic blood pressure; DBP, } \\
\text { diastolic blood pressure; WC, waist circumference; BMI, body mass index; FBS, Fasting blood sugar; } \\
\text { HbA1c; hemoglobin A1c; GGT, gamma-glutamyl transferase; UA, uric acid; Cr, creatinine; hs-CRP, highly } \\
\text { sensitive C-reactive protein; TC, total cholesterol; HDL-C, high density lipoprotein cholesterol; TG, } \\
\text { triglyceride; ALT, alanine aminotransferase; AST, aspartate amino transferase; LDL-C, low density } \\
\text { lipoprotein cholesterol; WBC, white blood cell; Hb, hemoglobin; Vit D, vitamin D; FLI, fatty liver index. }\end{array}$} \\
\hline \multicolumn{5}{|c|}{ Data are expressed as number (percentage) or mean \pm standard deviation. } \\
\hline The p-value was & nined through & are test and in & nt t-test. & \\
\hline
\end{tabular}

Comparison between variables for MAFLD prediction

The AUROC of FLI for the prediction of MAFLD was $0.776(95 \% \mathrm{Cl}, 0.737-0.816)$. The AUROC of FLI was higher than that of ALT $(0.694 ; 95 \% \mathrm{Cl}, 0.65-0.739)$, $\mathrm{Y}-\mathrm{GT}(0.701 ; 95 \% \mathrm{Cl}, 0.657-0.745)$, hsCRP $(0.620$; $95 \% \mathrm{Cl}, 0.572-0.668), \mathrm{Hb}(0.682 ; 95 \% \mathrm{Cl}, 0.635-0.729)$, and insulin $(0.717 ; 95 \% \mathrm{Cl}, 0.672-0.761)$ (all $\mathrm{P}<$ 0.0001 ; Fig. 1). The cut-off value of FLI for predicting MAFLD was 30.1037 , the sensitivity was $71.2 \%$, and the specificity was $71.3 \%$.

Concordance of MAFLD diagnosis using FLI and CT scans

We compared the correlation between the predicted MAFLD using FLI and the diagnosis of MAFLD using CT scans (Table 2). The correlation between the two was 30.1037 , which is the cut-off value of FLI obtained through the ROC curve. The results were consistent with $71.2 \%$ sensitivity and were statistically significant.

Table 2

Comparison between FLI for prediction of MAFLD.

\begin{tabular}{|c|c|c|c|c|}
\hline & & MAFLD = No & MAFLD = Yes & p \\
\hline \multirow[t]{2}{*}{ FLI } & No & 409(71.3) & $80(28.8)$ & \multirow[t]{2}{*}{$<0.0001$} \\
\hline & Yes & $165(28.7)$ & 198(71.2) & \\
\hline
\end{tabular}

\section{Discussion}


In our study, after analyzing the association between diagnosed MAFLD and abdominal CT, the sensitivity and specificity of FLI were 0.712 and 0.713 , respectively, with a cut-off value of 30.1037 .

MAFLD was previously known as NAFLD. The prevalence of MAFLD is increasing in Western countries owing to socioeconomic changes and the rapid transition from malnutrition to excess calorie eating habits. It is also increasing in many parts of the Asia-Pacific region, causing public health problems. Nutritionally unbalanced and unhealthy diet and excessive energy intake compared to expenditure contribute to the accumulation of triglycerides in the adipose tissue and liver. Particularly, Asians, are more likely to have visceral fat accumulation despite their low BMI [14]. South Asians in the United States have higher insulin resistance than Caucasians despite having the same or lower BMI [15]. Even nonobese and skinny Asians with NAFLD are at high risk for metabolic syndrome and type 2 diabetes mellitus $[14,16]$. Larger WC and visceral adipose tissue correlated more significantly with insulin resistance and NAFLD than with a higher BMI [17]. However, although WC and visceral adipose tissue can predict MAFLD, more accurate indicators should be developed. According to the Asian Pacific Association for the Study of the Liver Clinical Practice Guidelines, abdominal ultrasound is generally sufficient to detect hepatic steatosis and is the recommended primary diagnostic method for MAFLD imaging. Where possible, the measurement of controlled damping parameters using vibration-controlled transient elastography can be used as a more sensitive tool [6].

To evaluate NAFLD, a biopsy is performed. However, because it is invasive, a variety of imaging methods are increasingly being used. Imaging methods include both traditional and state-of-the-art technologies. Conventional imaging methods include B-mode ultrasound, CT, and magnetic resonance imaging. The diagnostic findings of patients with NAFLD using these imaging methods are based on lipid accumulation. Conventional imaging techniques cannot assess the degree of inflammation or fibrosis. Therefore, new imaging techniques have been developed, namely, ultrasonic elastography, quantitative ultrasound-based technology, magnetic resonance elastography, and magnetic resonance-based fat quantification technology $[18,19]$. In our study, fatty liver was confirmed using CT because it was only necessary to check lipid accumulation. WC was calculated using CT data to reduce WC measurement errors according to the examiner. If all imaging modalities are not available, such as in very large epidemiological studies, FLI can be used as an alternative method for diagnosing steatosis [6].

The sensitivity and specificity of FLI for diagnosing fatty liver at a cut-off of 60 in the original study were $61 \%$ and $86 \%$, respectively [7]. A population-based European study validated FLI in 2,652 elderly participants; it was demonstrated that FLI has a good predictive value in older people for both NAFLD in the population of any age as well as fatty liver diagnosed with ultrasound. The sensitivity and specificity for fatty liver were $62 \%$ and $81 \%$, respectively. For NAFLD prediction, the AUROC was 0.813 [8]. FLI has also been used to screen individuals at risk for NAFLD. FLI as a continuous measurement is closely related to the presence and severity of NAFLD, as assessed using ultrasonography [8]. FLI has been shown to be associated with cardiovascular morbidity, mortality, and diabetes incidence [20]. 
The 15-year all-cause, hepatic-related, cardiovascular disease (CVD)-related, and cancer-related mortality rates were obtained to determine whether FLI was associated with prognosis in a population study. FLI was independently associated with liver-related mortality and was associated with all-cause mortality, CVD-related mortality, and cancer-related mortality. These associations appear to be closely related to the risks posed by the insulin resistance correlation [20]. In a study of middle-aged non-diabetic patients, intima-media thickness, increased risk of CVD, and decreased insulin sensitivity were associated with high FLI values [21]. There is a French general population study of non-diabetic men and women aged 30 to 65 years. They analyzed the predictability of FLI and NAFLD fatty liver scores, two fatty liver markers for diabetes development. The use of logistic regression analysis to look at the categories of the FLI index and the odds ratio for the onset of diabetes demonstrated the usefulness of FLI [22]. Another Chinese study analyzed the accuracy and optimal cutoff point of FLI for predicting NAFLD in middle-aged and elderly people. In this cross-sectional study, liver ultrasonography was used to diagnose NAFLD and each AUROC curve and maximum Youden index analysis were used. As a result of that study, FLI was also able to accurately describe NAFLD, and the optimal cutoff point for middle-aged and elderly Chinese was 30 [11].

Jiang et al. conducted a study with 574 Chinese individuals to investigate whether FLI correlates with NAFLD and newly diagnosed coronary artery disease in a special Chinese population who underwent coronary angiography. FLI showed statistically significant results in predicting NAFLD; however, the AUROC was 0.721 , which was not effective than a BMI of 0.728 [23]. A Korean study verified the predictability of FLI in 376 patients. The results showed that the BMI's AUROC was the highest $(0.813)$, followed by that of WC (0.787) and FLI (0.785) [24]. The study targeted NAFLD, and the number of participants in our study was 2.3 times that of the previous study.

Previous studies have shown that low vitamin D levels are associated with NAFLD regardless of factors such as metabolic syndrome $[25,26]$. An Italian study conducted at the Center for Diabetes and Metabolic Diseases found that low vitamin D levels were associated with the presence of NAFLD regardless of the presence or absence of metabolic syndrome, diabetes and insulin resistance [25]. However, in our study, there was no significant relationship between vitamin D and MAFLD. Vitamin D regulates the metabolism of free fatty acids by reducing insulin resistance in peripheral tissues and liver cells. Vitamin D deficiency can accelerate the increase in serum free fatty acids, promote fat storage in the liver and lead to worsening of NAFLD [27]. If a larger-scale study is conducted that is more advanced than ours, it may lead to different results than this one. Our study has several limitations. First, it was conducted at a single center in a general hospital; hence, it cannot represent all Korean data. However, this limitation was supplemented using data from a larger number of participants compared to the previous study. Second, the diagnosis of fatty liver was not confirmed histologically through biopsy but was determined using CT.

In conclusion, our study verified the accuracy of FLI for predicting MAFLD using CT, and the AUROC of FLI for the prediction of MAFLD was 0.776 . This suggests that FLI can be used as a simple and cost-effective tool for screening MAFLD in clinical settings. In the future, FLI validation for predicting MAFLD should be performed in a large research group, and comparative analysis with other indices should be performed. 


\section{Abbreviations}

FLI, fatty liver index; ALT, alanine aminotransferase; GGT, gamma-glutamyl transferase; hs-CRP, highly sensitive C-reactive protein; $\mathrm{Hb}$, hemoglobin

\section{Declarations}

\section{Ethics approval and consent to participate}

This study followed the ethical standards laid out in the Declaration of Helsinki. The study was approved by the Clinical Trial Screening Committee of Wonkwang University Hospital (institutional review board [IRB] approval number: 2020-06-002-002).

The name of the IRB is Wonkwang University Hospital Institutional Review Board, which belongs to Wonkwang University 3rd General Hospital, and its address is as follows: Wonkwang University Hospital, Sinyong-dong 344-2, Iksan, Jeollabuk-do.

Consent was not required from all participants. We only reviewed patient charts for this study, we pledged that we would not use the information for anything other than research purposes. Also we reviewed the chart, which provided only the number of participants instead of their names. This was approved by the IRB.

\section{Consent to Publish}

Not applicable. This is because all patient information was investigated anonymously, and the manuscript did not reveal the patient's personal clinical information or the patient's image.

\section{Availability of data and material}

Not applicable.

\section{Competing interests}

The authors declares no competing interests.

\section{Author's contributions}

A Lum Han have made substantial contributions to the conception of the work; have drafted the work or substantively revised it.

Youngjon Kim have made interpretation of data.

A Lum Han approved the submitted version (and any substantially modified version that involves the author's contribution to the study); 
A Lum Han andYoungjon Kim have agreed both to be personally accountable for the author's own contributions and to ensure that questions related to the accuracy or integrity of any part of the work, even ones in which the author was not personally involved, are appropriately investigated, resolved, and the resolution documented in the literature.

\section{Funding}

This study was supported by Wonkwang University (2020).When this manuscript is published, researchers will receive a certain amount of research funding from Wonkwang University.

\section{Acknowledgements}

We thanksEditage, a professional English editing service, for editing this manuscript.

\section{References}

1. Eslam, M., et al., MAFLD: A Consensus-Driven Proposed Nomenclature for Metabolic Associated Fatty Liver Disease. Gastroenterology, 2020. 158(7): p. 1999-2014.e1.

2. Loomba, R. and A.J. Sanyal, The global NAFLD epidemic. Nature reviews Gastroenterology \& hepatology, 2013. 10(11): p. 686-690.

3. Tilg, H. and M. Effenberger, From NAFLD to MAFLD: when pathophysiology succeeds. Nature Reviews Gastroenterology \& Hepatology, 2020. 17(7): p. 387-388.

4. Fouad, Y., et al., The NAFLD-MAFLD debate: Eminence vs evidence. Liver International, 2021. 41(2): p. 255-260.

5. Lonardo, A., et al., Epidemiological modifiers of non-alcoholic fatty liver disease: Focus on high-risk groups. Digestive and Liver Disease, 2015. 47(12): p. 997-1006.

6. Eslam, M., et al., The Asian Pacific Association for the Study of the Liver clinical practice guidelines for the diagnosis and management of metabolic associated fatty liver disease. Hepatology international, 2020: p. 1-31.

7. Bedogni, G., et al., The Fatty Liver Index: a simple and accurate predictor of hepatic steatosis in the general population. BMC gastroenterology, 2006. 6(1): p. 1-7.

8. Koehler, E.M., et al., External validation of the fatty liver index for identifying nonalcoholic fatty liver disease in a population-based study. Clinical Gastroenterology and Hepatology, 2013. 11(9): p. 12011204.

9. Meffert, P.J., et al., Development, external validation, and comparative assessment of a new diagnostic score for hepatic steatosis. American Journal of Gastroenterology, 2014. 109(9): p. 14041414.

10. Yang, B.-L., et al., External validation of fatty liver index for identifying ultrasonographic fatty liver in a large-scale cross-sectional study in Taiwan. PloS one, 2015. 10(3): p. e0120443. 
11. Huang, X., et al., Validation of the fatty liver index for nonalcoholic fatty liver disease in middle-aged and elderly Chinese. Medicine, 2015. 94(40).

12. Hashimoto, E., M. Taniai, and K. Tokushige, Characteristics and diagnosis of NAFLD/NASH. Journal of gastroenterology and hepatology, 2013. 28: p. 64-70.

13. Motamed, N., et al., Fatty liver index vs waist circumference for predicting non-alcoholic fatty liver disease. World journal of gastroenterology, 2016. 22(10): p. 3023.

14. Lim, U., et al., Asian women have greater abdominal and visceral adiposity than Caucasian women with similar body mass index. Nutrition \& diabetes, 2011. 1(5): p. e6-e6.

15. Chandalia, M., et al., Insulin resistance and body fat distribution in South Asian men compared to Caucasian men. PloS one, 2007. 2(8): p. e812.

16. Fracanzani, A.L., et al., Liver and cardiovascular damage in patients with lean nonalcoholic fatty liver disease, and association with visceral obesity. Clinical Gastroenterology and Hepatology, 2017. 15(10): p. 1604-1611. e1.

17. Kuk, J.L., et al., Measurement site and the association between visceral and abdominal subcutaneous adipose tissue with metabolic risk in women. Obesity, 2010. 18(7): p. 1336-1340.

18. Pickhardt, P.J., et al., Specificity of unenhanced CT for non-invasive diagnosis of hepatic steatosis: implications for the investigation of the natural history of incidental steatosis. European radiology, 2012. 22(5): p. 1075-1082.

19. Fargion, S., M. Porzio, and A.L. Fracanzani, Nonalcoholic fatty liver disease and vascular disease: state-of-the-art. World Journal of Gastroenterology: WJG, 2014. 20(37): p. 13306.

20. Calori, G., et al., Fatty liver index and mortality: the Cremona study in the 15th year of follow-up. Hepatology, 2011. 54(1): p. 145-152.

21. Gastaldelli, A., et al., Fatty liver is associated with insulin resistance, risk of coronary heart disease, and early atherosclerosis in a large European population. Hepatology, 2009. 49(5): p. 1537-1544.

22. Balkau, B., et al., Nine-year incident diabetes is predicted by fatty liver indices: the French DESIR study. BMC gastroenterology, 2010. 10(1): p. 1-9.

23. Jiang, Z.-Y., et al., Fatty liver index correlates with non-alcoholic fatty liver disease, but not with newly diagnosed coronary artery atherosclerotic disease in Chinese patients. BMC gastroenterology, 2013. 13(1): p. 1-6.

24. Kim, J.H., et al., Validation of fatty liver index and lipid accumulation product for predicting fatty liver in Korean population. Liver international: official journal of the International Association for the Study of the Liver, 2011. 31(10): p. 1600-1601.

25. Barchetta, l., et al., Strong association between non alcoholic fatty liver disease (NAFLD) and low 25 $(\mathrm{OH})$ vitamin D levels in an adult population with normal serum liver enzymes. BMC medicine, 2011. 9(1): p. 1-7.

26. Seo, J.A., et al., Low vitamin D status is associated with nonalcoholic Fatty liver disease independent of visceral obesity in Korean adults. PLoS One, 2013. 8(10): p. e75197. 
27. Zhou, Q.G., et al., 1, 25-Dihydroxyvitamin D improved the free fatty-acid-induced insulin resistance in cultured C2C12 cells. Diabetes/metabolism research and reviews, 2008. 24(6): p. 459-464.

\section{Figures}

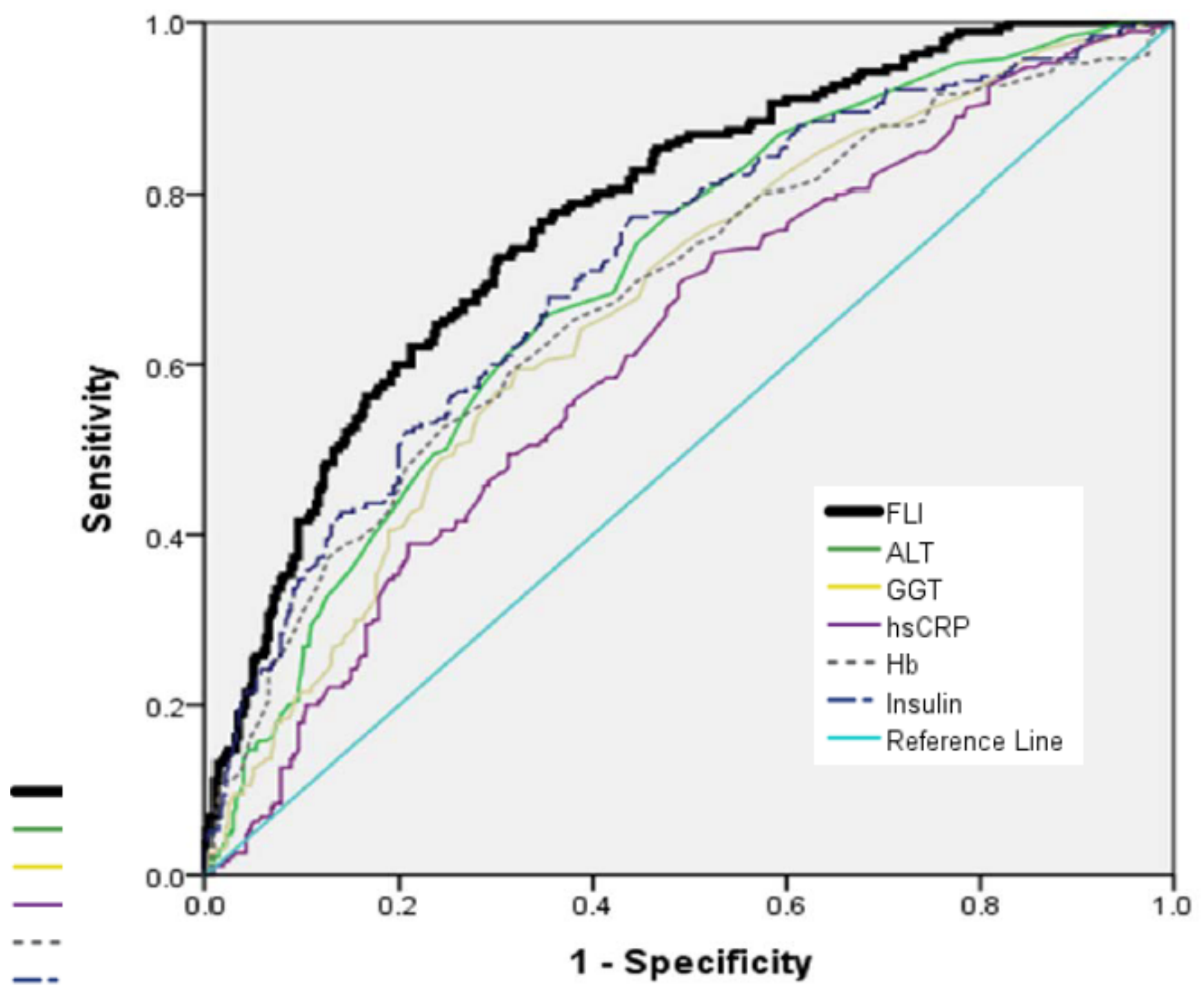

- Reference Line

Figure 1

ROC curves for FLI, ALT (IU/L), GGT (IU/L), hsCRP, Hb (g/dL), Insulin ( $\mu \mathrm{IU} / \mathrm{mL})$. FLI performed better than any single variable. (All P-values $<0.0001$ ) Abbreviations: FLI, fatty liver index; ALT, alanine aminotransferase; GGT, gamma-glutamyl transferase; hs-CRP, highly sensitive C-reactive protein; $\mathrm{Hb}$, hemoglobin 